\title{
PUBLIC HEALTH
}

\section{Vectorial Capacity of Triatoma guasayana (Wygodzinsky \& Abalos) (Hemiptera: Reduviidae) Compared with Two other Species of Epidemic Importance}

\author{
Manuel Loza-Murguía ${ }^{1}$, François Noireau ${ }^{2}$ \\ ${ }^{1}$ Department of Parasitology \& Microbiology, Unit Entomology, Academic Rural Unit Carmen Pampa, \\ Catholic Bolivian University San Pablo Coroico - Nor Yungas - La Paz, Bolivia, 5912 2137293; \\ boliviamanloza@yahoo.com \\ ${ }^{2}$ Institut Français de Recherche Scientifique pour le Développement en Coopération (ORSTOM-IRD), CP 9214, \\ La Paz, Bolivia \\ Edited by Eunice A B Galati - FSP/USP
}

Neotropical Entomology 39(5):799-809 (2010)

\begin{abstract}
Triatoma guasayana (Wygodzinsky \& Abalos) is a peridomestic triatomine with epidemiological importance in Bolivia, that may play an important role in the transmission of Trypanosoma cruzi (Chagas). In this study, two parameters of vectorial capacity were evaluated: the interval of feeding-defecation time and metacyclogenesis, in adult males and females and nymphal instars II to V of T. guasayana with comparisons with Triatoma infestans (Klug) and T. sordida (Stal). The results showed a close relationship between ingestion of blood and beginning of defecation. Values were negative in $T$. infestans, and $T$. sordida for instars II, III, IV, and V and also males and females but were positive in female $T$. sordida. Triatoma guasayana showed only negative values for instar II. Adults and nymphs began defecation as soon as they had finished feeding and required an average of $29.8 \mathrm{~min}$. The analysis of metacyclogenesis showed that T. guasayana was superior to T. infestans and $T$. sordida. However, the vectorial effectiveness of $T$. guasayana was significantly affected regarding the percentage of metacyclic trypomastigotes during instars III to $\mathrm{V}$ and showed a progressive increase. Females had higher proportions despite their ingestion being half that of $T$. infestans. The different instars of $T$. guasayana had a higher parasitic load than those of $T$. sordida and, although ingestion was $1 / 3$ of that of female $T$. infestans, there was a progressive increase in metacyclic trypomastigotes in the different nymphal stages of $T$. guasayana that decreased in adults.
\end{abstract}

KEY WORDS: Metacyclogenesis, feeding time, attacking time, feeding-defecation interval, Chrono M

In 1991, Southern Cone countries initiated a project whose main objective was to stop vectorial transmission of Chagas disease by Triatoma infestans (Klug) and also by blood transfusion in Argentina, Brazil, Bolivia, Chile, Paraguay and Uruguay. (Schofield et al 2006, OMS 2007). Results showed that the Health Ministries of Latin-American countries had efficient and economically viable methods for establishing vectorial control and blood transfusion programs which would permit the elimination of some vector species (OMS 2007). However, this was at odds with the recent increase of cases of Chagas disease in the Gran Chaco of Argentina (Gürtler et al 2005).

At the moment, 141 species of Triatominae are described in the New World (Galvão et al 2003, Galvão \& Angulo 2006, Costa et al 2006, Bérenger \& Blanchet 2007, Costa \& Félix 2007, Martinez et al 2007, Sandoval et al 2007). The reduction in T. infestans (Klug) populations in residences has created an available niche for other vectors and the tendency for peridomestic or wild species to occupy these available niches in Paraguay and Brazil has been demonstrated (Dias
1988, Ruas Neto \& Krug 1995, Rojas-de-Arias 1996). In Uruguay, Rosa \& Salvatella (1995) warned of the presence of Triatoma rubrovaria ((Blanchard) in residences, and Bar et al (1993) and Wisnivesky-Colli et al (1993) demonstrated the potential risk that Triatoma sordida (Stal) and Triatoma guasayana (Wygodzinsky \& Abalos) represented in Argentina. Triatoma sordida and T. guasayana are closely related species of Triatominae since they are morphologically similar and occupy the same ecotopes with an overlapping distribution in northern Argentina and part of the Bolivian Chaco and Paraguay (Schofield 1994,Usinger et al 1966). Although originally they were wild species, they were frequently found in peridomestic and domestic habitats (Forattini 1980, Wisnivesky-Colli et al 1993, Gajate et al 1996, Noireau et al 1998) due to their domestic tendency, and may be considered as possible domestic vectors of Chagas disease.

Triatoma sordida and T. guasayana both occur in Bolivia (Noireau et al 1999), and have adapted to the human environment but they are also found in forest habitats. Both 
these species might be potential vectors since they frequently substitute $T$. infestans after control operations. In the forest environment, T. sordida and T. guasayana were found in a variety of ecotopes: bird nests, tree holes, under bark, trees roots, palm trees, in groups of bromeliads and under rocks (Barretto 1971, Forattini et al 1971, Carcavallo \& Martinez 1985, Diotaiuti et al 1993). In the Bolivian Chaco, these species were mainly collected from trees holes and bromeliads (Noireau et al 2000) and the adults have a high dispersal potential by flying at night during the hot, dry months (August-November) (Wisnivesky-Colli et al 1993, Noireau et al 1998). Studies on triatomine species have demonstrated that hunger is the main trigger for starting flight (Sjogren \& Ryckman 1996, Ekkens 1981, Lehane \& Schofield, 1982, McEwen \& Lehane 1993) and, therefore, nutritional condition might indicate whether adults will fly or not. (Lehane \& Schofield 1982).

Triatoma guasayana has high populations in the wild, mainly in biotopes that include Cactacea (Opuntia quimilo), chaguares (Bromelia sp.) and trunks and this species usually feeds on rodents, marsupials and birds (Carcavallo et al 1988, Vezzani et al 2001). Its epidemiological importance is considered but in 1985 its presence in housing, where $T$. infestans had been controlled by insecticides (Carcavallo et $a l, 1985)$, was mentioned. Studies in Argentina showed that both this species and $T$. sordida were secondary vectors of Trypanosoma cruzi (Chagas) and would be a potential danger for human beings during entomological surveillance, since $T$. guasayana, is not only able to invade homes and attack human beings and dogs but has been found naturally infected with the parasite (Carcavallo et al 1985, Wisnivesky-Colli et al 1993, Gajate et al 1996, Cecere 1999, Canale 2000).

In order to be an efficient vector of T. cruzi, a Triatominae species must have the following characteristics (Lent \& Wygodzinsky 1979): i) inhabit dwellings and have a high reproductive rate; ii) be susceptible to infection by $T$. cruzi (infection rate by $T$. cruzi: high and efficient metacyclogenesis); iii) be anthropophylic and have a short feeding-defecation interval.

With the objective of evaluating the potential epidemiological importance of T. guasayana and T. sordida, two important parameters which control the vectorial capacity of both species were studied: the feeding-defecation process and their metacyclogenesis, with comparisons made with the main vector, $T$. infestans. These data may predict differences between the abilities of these species as vectors (Mello 1980, Kirk \& Schofield 1987, Trumper \& Gorla 1991, Crocco \& Catalá 1996).

\section{Material and Methods}

The nymphal instars and adult males and females were supplied by the Insectary of the Instituto Boliviano de Biología de la Altura (I.B.B.A.) from fifth generation triatomine colonies. The insects were kept in the laboratory at $28 \pm 2^{\circ} \mathrm{C}$ and $60-70 \%$ RH. The Tulahuén strain of T. cruzi was provided by the Phytochemistry Department of I.B.B.A and the female Swiss mice were supplied by the Laboratory of the Animals Center of the Instituto de Laboratorios de Salud
(INLASA). All the research was carried out at the Tropical Diseases Laboratory of I.B.B.A.

Feeding/defecation study. An apparatus called Chrono $\mathrm{M}$, designed by Noireau (unpublished data), was used to determine the feeding-defecation interval of the triatomines (Fig 1). Chrono $\mathrm{M}$ is a plastic container of $250 \mathrm{~cm}^{3}$, which has a millimeter mesh in the upper part that puts the mouse in contact with the food source. Both the upper and lower parts have electronic detectors which are activated when the insect is in contact with the food source and are deactivated when the insect deposits its feces. Chrono M has a digital chronometer which measures time in seconds. This apparatus was used for $5^{\text {th }}$ instar nymphs and adults but the $2^{\text {nd }}, 3^{\text {rd }}$ and $4^{\text {th }}$ nymphal instars were observed directly using an ordinary chronometer.

Insects. Triatoma sordida of Cotoca from Santa Cruz (216 insects), T. guasayana of Izozog from Santa Cruz (180) and T. infestans of Izozog from Santa Cruz (173) as the control. After two successive meals, the insects were starved for 30 days and the following parameters were evaluated: (i) time of attack or time interval between placing the insect in the container and its contact with the mouse, with the penetration of the rostrum into the host tegument; (ii) feeding time or time interval between the penetration of the insect rostrum into the mouse tegument and its withdrawal after finishing blood ingestion; (iii) defecation time or time interval between the penetration of the insect rostrum into the mouse tegument and excretion of the first drop of urine and/or feces. (iv) the determination of the time interval for feeding-defecation, which is the difference between

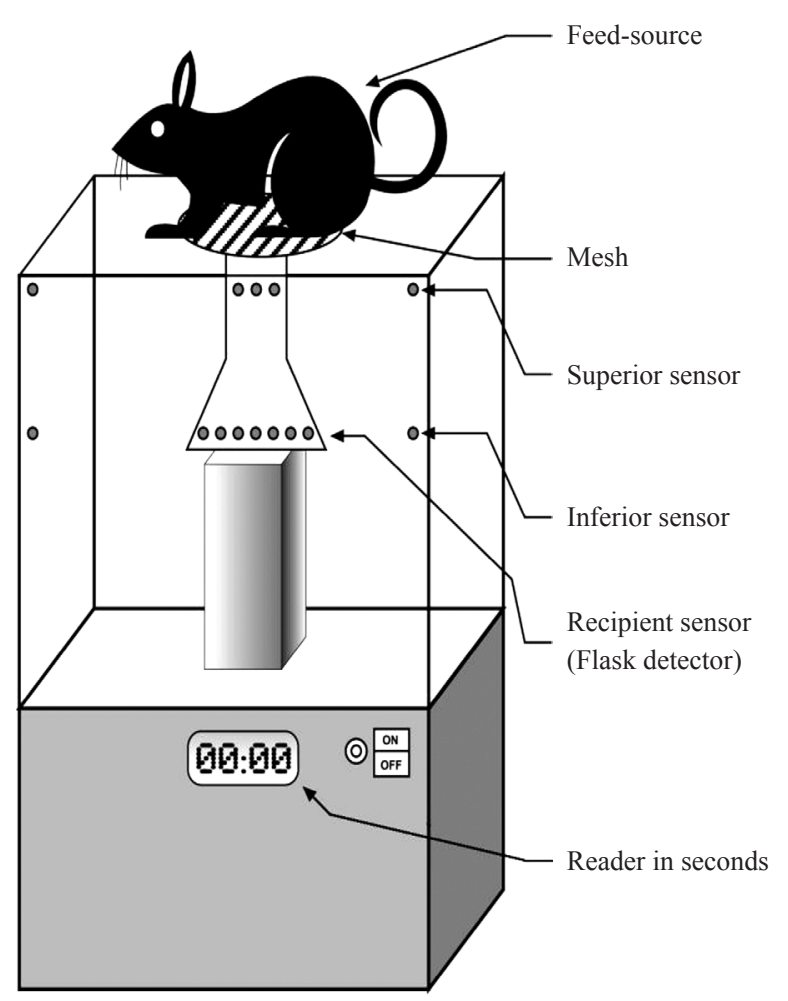

Fig 1 Chrono $\mathrm{M}$. 
the feeding and defecation times. If this interval was negative, the insect defecated before finishing blood feeding.

Metacyclogenesis. This is the process of transformation of the epimastigote forms of $T$. cruzi into metacyclic trypomastigote forms which takes place in the triatomine alimentary canal. Six female Swiss mice were infected intraperitoneally with an inoculation of $100 \mu \mathrm{l}$ of the Tulahuén strain of T. cruzi from a culture that contained $2.410^{6}$ parasites $/ \mathrm{cm}^{3}$. Nymphs of the $3^{\text {rd }}$, $4^{\text {th }}$ and $5^{\text {th }}$ instars, as well as adults of $T$. sordida (39 insects), $T$. guasayana (40 insects) and T. infestans (33 insects), were used for evaluating metacyclogenesis. The insects were starved for thirty days before feeding them for $30 \mathrm{~min}$ on infected mice. After 30 days, their abdomens were squeezed to obtain feces and if preliminary observations showed the presence of flagellates, $50 \mu \mathrm{l}$ of their faeces in two or three drops of physiologic solution were centrifuged at $1500 \mathrm{rpm}$ for $3 \mathrm{~min}$ in saline solution. The sediment was treated with a mixture of absolute ethanol and giemsa and observations were made using an Olympus $\mathrm{CH} 30$ microscope with an immersion objective (100x) and the counts made over 200 fields.

Statistical methods. Means were compared using the Student t test (when one of the samples $<30$ ) and reduced deviation or R.D. (when both samples $>30$ )

\section{Results}

\section{Feeding - defecation study}

Attacking time. Triatoma infestans had the shortest attacking times; T. sordida needed less time for starting the feeding process than $T$. guasayana, for males and for the $3^{\text {rd }}$ and $4^{\text {th }}$ nymphal instars. The differences between both species were not significant for the other instars (Table 1).

Feeding time. Triatoma infestans fed faster than T. guasayana and $T$. sordida, which fed for longer during all the nymphal instars, except for the $5^{\text {th }}$ instar where there were no significant differences (Table 2).

Defecation time. Triatoma infestans defecated faster for all the instars, followed by $T$. sordida and $T$. guasayana and all the differences were significant (Table 3).

Time interval of feeding-defecation. Except for the $2^{\text {nd }}$ instar, T. guasayana defecated after having finished feeding whereas the other two species defecated before withdrawing their rostrum from the host tissue. Triatoma sordida showed a significant advantage in the $1^{\text {st }}, 2^{\text {nd }}$ and $3^{\text {rd }}$ nymphal instars over $T$. infestans. A tendency to defecate rapidly was observed in these instars for both species, and especially in T. sordida (Table 4).

Metacyclogenesis. In the $6^{\text {th }}$ mice infected with $T$. cruzi, the highest peak of parasitemia was observed on the $7^{\text {th }}$ day and animals died on the $10^{\text {th }}$ day. The triatomines were infected on the $7^{\text {th }}$ day when the parasitemia had the highest peak (Table 5 ). No differences in parasite populations were observed among the different stages of the same species. Triatoma infestans had the biggest population of trypomastigotes (20.5 trypomastigotes in 200 fields vs. 15.4 for T. guasayana and 13.6 for $T$. sordida, R.D. $=(\mathrm{P}<0.001)$. The percentages of metacyclic trypomastigotes were not significantly different between the three species $(11.5 \%$ in $T$. infestans vs. $9.2 \%$ in the other two species; R.D. $=(\mathrm{P}<0.05)($ Table 6$)$.

\section{Discussion}

According to Noireau (1999), the vector candidates are triatomines found in the wild but that may occasionally invade artificial ecotopes where they establish small colonies. Various reports of $T$. sordida and $T$. guasayana in human environments in Southern Cone countries (Forattini et al 1982, Carcavallo et al 1988, Noireau et al 1997) make them vector candidates and this should encourage more studies on these species. With regard to the vectorial control of Chagas disease, the peridomicile vectors have a special importance due to their role in these processes and the re-infestation of housing during entomological surveillance. Among these vectors, $T$. guasayana and $T$. sordida, are species which might play a significant role in the transmission of $T$. cruzi to human beings, specially after the eradication of $T$. infestans from houses (Carcavallo et al 1985, Wisnivesky-Colli et al 1993, Gajate et al 1996, Cecere et al 1999, Noireau et al 1999, Canale 2000).

According to Wisnivesky-Colli et al (1993), T. guasayana would develop bigger populations in a domiciliary environment. It is clear that domiciliary transmission of $T$. cruzi depends on factors, such as the population size of triatomines, which has to reach a critical size (Noireau et al 1997). For this reason, from just considering the population dynamics, it is improbable that any of these species might be as efficient a vector as T. infestans (Schofield 1994). However, it is known that this biological criterion is not the only one and others, such as the process of feeding-defecation and metacyclogenesis of T. cruzi, are also very important (OMS 1991).

The dependence of defecation time on the quantity of ingested blood was observed in T. infestans (Trumper \& Gorla 1991) and Rhodnius prolixus (Kirk \& Schofield 1987); these two variables show a similarity in the feedingdefecation interval. A minimum blood feeding of $80 \mathrm{mg}$ was enough for defecations to occur during feeding by $5^{\text {th }}$ instar nymphs, while nymphs of the $5^{\text {th }}$ instar of $T$. infestans, which fed on the same quantity of blood, had a feeding-defecation interval of 15 min after feeding (Trumper \& Gorla 1991). Comparison of these two parameters between T. infestans and $T$. sordida show that adult females of $T$. sordida are more efficient when defecating compared to the other instars (Perlowagora 1973, Zeledon et al 1977). The $5^{\text {th }}$ nymphal instar of $T$. sordida feeds on more blood than $T$. infestans, which allows this species to survive longer with just one meal (Perlowagora 1973, Juarez \& Castro 1982). Nymphs and adults of $T$. sordida defecated immediately after feeding or frequently defecated during feeding (Crocco \& Catalá 1996); the quantity ingested depended on the nutritional state of insect at the moment of feeding, which is related to dispersal activity. Different studies have shown that a low nutritional state is a determinant for adult dispersal by flying 
Table 1 Mean time (min) for beginning an attack at the blood source, comparision of the different vectors and different instars (Triatoma infestans, T. sordida, T. guasayana).

\begin{tabular}{|c|c|c|c|c|c|c|c|c|c|}
\hline Vector & Instar & No & Min. & Max. & Mean \pm SD & DM & $\mathrm{P}<$ & $\mathrm{t}$ & $\mathrm{P}<$ \\
\hline T. sordida & II & 31 & 0.60 & 2.20 & $1.40 \pm 0.80$ & 0.77 & N.S. & & \\
\hline T. guasayana & II & 32 & 0.80 & 2.40 & $1.60 \pm 0.80^{\mathrm{a}}$ & & & & \\
\hline T. sordida & II & 31 & 0.60 & 2.20 & $1.40 \pm 0.80$ & 3.19 & 0.01 & & \\
\hline T. infestans & II & 36 & 0.40 & 1.40 & $0.90 \pm 0.50$ & & & & \\
\hline T. guasayana & II & 32 & 0.80 & 2.40 & $1.60 \pm 0.80^{\mathrm{a}}$ & 4.27 & 0.001 & & \\
\hline T. infestans & II & 36 & 0.40 & 1.40 & $0.90 \pm 0.50$ & & & & \\
\hline T. sordida & III & 40 & 0.10 & 1.50 & $0.80 \pm 0.70$ & 5.58 & 0.001 & & \\
\hline T. guasayana & III & 42 & 0.80 & 2.80 & $1.80 \pm 1.00^{\mathrm{a}}$ & & & & \\
\hline T. sordida & III & 40 & 0.10 & 1.50 & $0.80 \pm 0.70$ & 2.12 & 0.05 & & \\
\hline T. infestans & III & 39 & 0.10 & 0.90 & $0.50 \pm 0.40$ & & & & \\
\hline T. guasayana & III & 42 & 0.80 & 2.80 & $1.80 \pm 1.00^{\mathrm{a}}$ & 8.34 & 0.001 & & \\
\hline T. infestans & III & 39 & 0.10 & 0.90 & $0.50 \pm 0.40$ & & & & \\
\hline T. sordida & IV & 44 & 0.10 & 1.50 & $0.70 \pm 0.80$ & 5.59 & 0.001 & & \\
\hline T. guasayana & IV & 44 & 0.80 & 2.40 & $1.60 \pm 0.80^{\mathrm{a}}$ & & & & \\
\hline T. sordida & IV & 44 & 0.10 & 1.50 & $0.70 \pm 0.80$ & 1.54 & N.S. & & \\
\hline T. infestans & IV & 43 & 0.20 & 0.80 & $0.50 \pm 0.30$ & & & & \\
\hline T. guasayana & IV & 44 & 0.80 & 2.40 & $1.60 \pm 0.80^{\mathrm{a}}$ & 8.61 & 0.001 & & \\
\hline T. infestans & IV & 43 & 0.20 & 0.80 & $0.50 \pm 0.30$ & & & & \\
\hline T. sordida & V & 61 & 0.30 & 0.11 & $0.60 \pm 0.30$ & 1.17 & N.S. & & \\
\hline T. guasayana & V & 64 & 0.30 & 1.60 & $0.70 \pm 0.60^{\mathrm{a}}$ & & & & \\
\hline T. sordida & V & 61 & 0.30 & 0.11 & $0.60 \pm 0.30$ & 1.55 & N.S. & & \\
\hline T. infestans & V & 64 & 0.20 & 0.99 & $0.50 \pm 0.30$ & & & & \\
\hline T. guasayana & V & 64 & 0.30 & 1.60 & $0.70 \pm 0.60^{\mathrm{a}}$ & 2.76 & 0.05 & & \\
\hline T. infestans & V & 64 & 0.20 & 0.99 & $0.50 \pm 0.30$ & & & & \\
\hline T. sordida & Males & 17 & 0.10 & 0.39 & $0.20 \pm 0.10$ & & & 5.12 & 0.001 \\
\hline T. guasayana & Males & 26 & 0.60 & 1.90 & $1.10 \pm 0.70^{\mathrm{a}}$ & & & & \\
\hline T. sordida & Males & 17 & 0.10 & 0.39 & $0.20 \pm 0.10$ & & & 1.69 & N.S. \\
\hline T. infestans & Males & 25 & 0.10 & 0.60 & $0.30 \pm 0.20$ & & & & \\
\hline T. guasayana & Males & 26 & 0.60 & 1.90 & $1.10 \pm 0.70^{\mathrm{a}}$ & & & 5.20 & 0.001 \\
\hline T. infestans & Males & 25 & 0.10 & 0.60 & $0.30 \pm 0.20$ & & & & \\
\hline T. sordida & Females & 23 & 0.10 & 0.40 & $0.30 \pm 0.20$ & & & 0.38 & N.S. \\
\hline T. guasayana & Females & 26 & 0.20 & 0.44 & $0.30 \pm 0.10^{\mathrm{a}}$ & & & & \\
\hline T. sordida & Females & 23 & 0.10 & 0.40 & $0.30 \pm 0.20$ & & & 0.21 & N.S. \\
\hline T. infestans & Females & 27 & 0.20 & 0.60 & $0.30 \pm 0.30$ & & & & \\
\hline T. guasayana & Females & 26 & 0.20 & 0.44 & $0.30 \pm 0.10^{\mathrm{a}}$ & & & 0.06 & N.S. \\
\hline T. infestans & Females & 27 & 0.20 & 0.60 & $0.30 \pm 0.30$ & & & & \\
\hline
\end{tabular}

No: number of insects; SD: standard deviation; a: test comparision of the mean: $\mathrm{DM}=$ reduced deviation $>30 ; \mathrm{t}=$ student test $<30$ 
Table 2 Mean feeding time (min) of instar at blood source for the three vectors and different instars (Triatoma infestans, T. sordida, T. guasayana).

\begin{tabular}{|c|c|c|c|c|c|c|c|c|c|}
\hline Vector & Instar & No & Min. & Max. & Mean \pm SD & $\mathrm{DM}$ & $\mathrm{P}<$ & $\mathrm{t}$ & $\mathrm{P}<$ \\
\hline T. sordida & II & 30 & 24.5 & 39.0 & $32.0 \pm 6.9$ & 2.30 & 0.05 & & \\
\hline T. guasayana & II & 30 & 24.5 & 32.0 & $28.7 \pm 3.7^{\mathrm{a}}$ & & & & \\
\hline T. sordida & II & 30 & 24.5 & 39.0 & $32.0 \pm 6.9$ & & & 4.00 & 0.001 \\
\hline T. infestans & II & 28 & 19.3 & 32.0 & $25.2 \pm 5.8$ & & & & \\
\hline T. guasayana & II & 30 & 24.5 & 32.0 & $28.7 \pm 3.7^{\mathrm{a}}$ & & & 2.72 & 0.01 \\
\hline T. infestans & II & 28 & 19.3 & 32.0 & $25.2 \pm 5.8$ & & & & \\
\hline T. sordida & III & 40 & 23.0 & 46.0 & $35.1 \pm 10.4$ & 5.05 & 0.001 & & \\
\hline T. guasayana & III & 37 & 20.0 & 30.0 & $25.9 \pm 4.7^{\mathrm{a}}$ & & & & \\
\hline T. sordida & III & 40 & 23.0 & 46.0 & $35.1 \pm 10.4$ & 7.99 & 0.001 & & \\
\hline T. infestans & III & 30 & 15.0 & 25.0 & $20.4 \pm 4.6$ & & & & \\
\hline T. guasayana & III & 37 & 20.0 & 30.0 & $25.9 \pm 4.7^{\mathrm{a}}$ & 4.89 & 0.001 & & \\
\hline T. infestans & III & 30 & 15.0 & 25.0 & $20.4 \pm 4.6$ & & & & \\
\hline T. sordida & IV & 40 & 20.5 & 45.0 & $33.3 \pm 11.5$ & 4.68 & 0.001 & & \\
\hline T. guasayana & IV & 32 & 21.0 & 28.5 & $24.3 \pm 3.5^{\mathrm{a}}$ & & & & \\
\hline T. sordida & IV & 40 & 20.5 & 45.0 & $33.3 \pm 11.5$ & 4.60 & 0.001 & & \\
\hline T. infestans & IV & 30 & 18.5 & 28.5 & $24.1 \pm 4.6$ & & & & \\
\hline T. guasayana & IV & 32 & 21.0 & 28.5 & $24.3 \pm 3.5^{\mathrm{a}}$ & 0.21 & N.S. & & \\
\hline T. infestans & IV & 30 & 18.5 & 28.5 & $24.1 \pm 4.6$ & & & & \\
\hline T. sordida & V & 28 & 21.9 & 42.5 & $31.8 \pm 9.3$ & & & 0.08 & N.S. \\
\hline T. guasayana & V & 29 & 29.0 & 34.0 & $31.6 \pm 2.1^{\mathrm{a}}$ & & & & \\
\hline T. sordida & V & 28 & 21.9 & 42.5 & $31.8 \pm 9.3$ & & & 2.97 & 0.01 \\
\hline T. infestans & V & 30 & 19.5 & 31.5 & $25.8 \pm 5.7$ & & & & \\
\hline T. guasayana & V & 29 & 29.0 & 34.0 & $31.6 \pm 2.1^{\mathrm{a}}$ & & & 5.15 & 0.001 \\
\hline T. infestans & V & 30 & 19.5 & 31.5 & $25.8 \pm 5.7$ & & & & \\
\hline T. sordida & Males & 28 & 25.5 & 40.0 & $33.2 \pm 6.5$ & & & 0.90 & N.S. \\
\hline T. guasayana & Males & 26 & 28.5 & 35.0 & $31.9 \pm 3.0^{\mathrm{a}}$ & & & & \\
\hline T. sordida & Males & 28 & 25.5 & 40.0 & $33.2 \pm 6.5$ & & & 2.62 & 0.01 \\
\hline T. infestans & Males & 34 & 21.5 & 34.6 & $28.9 \pm 6.2$ & & & & \\
\hline T. guasayana & Males & 26 & 28.5 & 35.0 & $31.9 \pm 3.0^{\mathrm{a}}$ & & & 1.47 & N.S. \\
\hline T. infestans & Males & 34 & 21.5 & 34.6 & $28.9 \pm 6.2$ & & & & \\
\hline T. sordida & Females & 29 & 26.0 & 35.0 & $31.4 \pm 4.2$ & & & 0.12 & N.S. \\
\hline T. guasayana & Females & 26 & 27.5 & 34.0 & $31.2 \pm 2.7^{\mathrm{a}}$ & & & & \\
\hline T. sordida & Females & 29 & 26.0 & 35.0 & $31.4 \pm 4.2$ & & & 5.93 & 0.001 \\
\hline T. infestans & Females & 21 & 17.5 & 28.0 & $23.5 \pm 5.1$ & & & & \\
\hline T. guasayana & Females & 26 & 27.5 & 34.0 & $31.2 \pm 2.7^{\mathrm{a}}$ & & & 6.64 & 0.001 \\
\hline T. infestans & Females & 21 & 17.5 & 28.0 & $23.5 \pm 5.1$ & & & & \\
\hline
\end{tabular}

No: number of insects; SD: standard deviation; a: test comparison of the mean: DM: reduced deviation $>30 ; \mathrm{t}=$ student test $<30$ 
Table 3 Mean defecation time (min) of different instars of Triatoma infestans, T. sordida, T. guasayana.

\begin{tabular}{|c|c|c|c|c|c|c|c|c|c|}
\hline Vector & Instar & No & Min. & Max. & Mean \pm DS & $\mathrm{DM}$ & $\mathrm{P}<$ & $\mathrm{t}$ & $\mathrm{P}<$ \\
\hline T. sordida & II & 31 & 18.9 & 25.0 & $21.8 \pm 2.4$ & 11.36 & 0.001 & & \\
\hline T. guasayana & II & 32 & 25.5 & 31.0 & $28.3 \pm 2.2^{\mathrm{a}}$ & & & & \\
\hline T. sordida & II & 31 & 18.9 & 25.0 & $21.8 \pm 2.4$ & 10.29 & 0.001 & & \\
\hline T. infestans & II & 36 & 12.5 & 19.0 & $15.6 \pm 2.5$ & & & & \\
\hline T. guasayana & II & 32 & 25.5 & 31.0 & $28.3 \pm 2.2^{\mathrm{a}}$ & 22.20 & 0.001 & & \\
\hline T. infestans & II & 36 & 12.5 & 19.0 & $15.6 \pm 2.5$ & & & & \\
\hline T. sordida & III & 39 & 18.8 & 25.7 & $22.9 \pm 3.3$ & 3.86 & 0.001 & & \\
\hline T. guasayana & III & 44 & 20.7 & 31.8 & $26.7 \pm 5.5^{\mathrm{a}}$ & & & & \\
\hline T. sordida & III & 39 & 18.8 & 25.7 & $22.9 \pm 3.3$ & 11.98 & 0.001 & & \\
\hline T. infestans & III & 39 & 11.6 & 16.9 & $15.2 \pm 2.3$ & & & & \\
\hline T. guasayana & III & 44 & 20.7 & 31.8 & $26.7 \pm 5.5^{\mathrm{a}}$ & 12.58 & 0.001 & & \\
\hline T. infestans & III & 39 & 11.6 & 16.9 & $15.2 \pm 2.3$ & & & & \\
\hline T. sordida & IV & 44 & 18.4 & 25.3 & $23.1 \pm 3.2$ & 6.17 & 0.001 & & \\
\hline T. guasayana & IV & 44 & 22.8 & 35.0 & $28.8 \pm 5.2^{\mathrm{a}}$ & & & & \\
\hline T. sordida & IV & 44 & 18.4 & 25.3 & $23.1 \pm 3.2$ & 6.13 & 0.001 & & \\
\hline T. infestans & IV & 44 & 15.9 & 20.8 & $19.6 \pm 2.3$ & & & & \\
\hline T. guasayana & IV & 44 & 22.8 & 35.0 & $28.8 \pm 5.2^{\mathrm{a}}$ & 10.81 & 0.001 & & \\
\hline T. infestans & IV & 44 & 15.9 & 20.8 & $19.6 \pm 2.3$ & & & & \\
\hline T. sordida & $\mathrm{V}$ & 61 & 25.0 & 35.0 & $30.3 \pm 4.4$ & 6.99 & 0.001 & & \\
\hline T. guasayana & $\mathrm{V}$ & 64 & 31.0 & 39.0 & $35.3 \pm 3.6^{\mathrm{a}}$ & & & & \\
\hline T. sordida & $\mathrm{V}$ & 61 & 25.0 & 35.0 & $30.3 \pm 4.4$ & 14.19 & 0.001 & & \\
\hline T. infestans & $\mathrm{V}$ & 64 & 16.9 & 23.0 & $20.7 \pm 2.9$ & & & & \\
\hline T. guasayana & $\mathrm{V}$ & 64 & 31.0 & 39.0 & $35.3 \pm 3.6^{\mathrm{a}}$ & 25.25 & 0.001 & & \\
\hline T. infestans & $\mathrm{V}$ & 64 & 16.9 & 23.0 & $20.7 \pm 2.9$ & & & & \\
\hline T. sordida & Males & 17 & 28.6 & 34.8 & $32.2 \pm 3.1$ & & & 2.75 & 0.01 \\
\hline T. guasayana & Males & 26 & 31.5 & 36.5 & $34.5 \pm 2.5^{\mathrm{a}}$ & & & & \\
\hline T. sordida & Males & 17 & 28.6 & 34.8 & $32.2 \pm 3.1$ & & & 5.29 & 0.001 \\
\hline T. infestans & Males & 25 & 19.5 & 28.0 & $25.0 \pm 4.9$ & & & & \\
\hline T. guasayana & Males & 26 & 31.5 & 36.5 & $34.5 \pm 2.5^{\mathrm{a}}$ & & & 8.71 & 0.001 \\
\hline T. infestans & Males & 25 & 19.5 & 28.0 & $25.0 \pm 4.9$ & & & & \\
\hline T. sordida & Females & 23 & 28.8 & 33.0 & $31.6 \pm 2.1$ & & & 3.59 & 0.001 \\
\hline T. guasayana & Females & 26 & 30.7 & 36.9 & $34.4 \pm 3.1^{\mathrm{a}}$ & & & & \\
\hline T. sordida & Females & 23 & 28.8 & 33.0 & $31.6 \pm 2.1$ & & & 7.73 & 0.001 \\
\hline T. infestans & Females & 27 & 17.0 & 28.0 & $22.6 \pm 5.3$ & & & & \\
\hline T. guasayana & Females & 26 & 30.7 & 36.9 & $34.4 \pm 3.1^{\mathrm{a}}$ & & & 9.87 & 0.001 \\
\hline T. infestans & Females & 27 & 17.0 & 28.0 & $22.6 \pm 5.3$ & & & & \\
\hline
\end{tabular}

No: number of insects; SD: standard deviation; a: test comparison of the mean: DM: reduced deviation $>30 ; \mathrm{t}=$ student test $<30$ 
Table 4 Mean feeding-defecation interval (min) for each instar of Triatoma infestans, T. sordida, T. guasayana.

\begin{tabular}{|c|c|c|c|c|c|}
\hline Vector & Instar & No & $\begin{array}{l}\text { Feeding time } \\
\text { Mean } \pm \text { SD }\end{array}$ & $\begin{array}{l}\text { Feeding-defecation } \\
\text { Mean } \pm \text { SD }\end{array}$ & $\begin{array}{c}\text { Feeding-defecation interval } \\
\text { Mean } \pm \text { SD }\end{array}$ \\
\hline T. sordida & II & 31 & $32.0 \pm 6.9$ & $21.8 \pm 2.4$ & $-10.2 \pm 4.50$ \\
\hline T. guasayana & II & 32 & $28.7 \pm 3.7$ & $28.3 \pm 2.2$ & $-0.40 \pm 1.50$ \\
\hline T. infestans & II & 36 & $25.2 \pm 5.8$ & $15.6 \pm 2.5$ & $-9.60 \pm 3.30$ \\
\hline T. sordida & III & 40 & $35.1 \pm 10.4$ & $22.9 \pm 3.3$ & $-12.2 \pm 7.10$ \\
\hline T. guasayana & III & 42 & $25.9 \pm 4.7$ & $26.7 \pm 5.5$ & $+0.70 \pm 0.80$ \\
\hline T. infestans & III & 32 & $20.4 \pm 4.7$ & $15.2 \pm 2.3$ & $-5.10 \pm 2.40$ \\
\hline T. sordida & IV & 44 & $33.3 \pm 11.5$ & $23.1 \pm 3.2$ & $-10.1 \pm 8.30$ \\
\hline T. guasayana & IV & 44 & $24.3 \pm 3.5$ & $28.8 \pm 5.2$ & $+4.60 \pm 1.70$ \\
\hline T. infestans & IV & 44 & $24.1 \pm 4.6$ & $19.6 \pm 2.3$ & $-4.50 \pm 2.30$ \\
\hline T. sordida & $\mathrm{V}$ & 61 & $31.8 \pm 9.3$ & $30.3 \pm 4.4$ & $-1.50 \pm 4.90$ \\
\hline T. guasayana & $\mathrm{V}$ & 64 & $31.6 \pm 2.1$ & $35.3 \pm 3.6$ & $+3.70 \pm 1.50$ \\
\hline T. infestans & V & 64 & $25.8 \pm 5.7$ & $20.7 \pm 2.9$ & $-5.10 \pm 2.80$ \\
\hline T. sordida & Males & 17 & $33.2 \pm 6.5$ & $32.2 \pm 3.1$ & $-1.00 \pm 3.40$ \\
\hline T. guasayana & Males & 26 & $31.9 \pm 3.0$ & $34.5 \pm 2.5$ & $+2.60 \pm 0.50$ \\
\hline T. infestans & Males & 25 & $28.9 \pm 6.2$ & $25.0 \pm 4.9$ & $-3.90 \pm 1.30$ \\
\hline T. sordida & Females & 23 & $31.4 \pm 4.2$ & $31.6 \pm 2.1$ & $+0.30 \pm 2.10$ \\
\hline T. guasayana & Females & 26 & $31.2 \pm 2.7$ & $34.4 \pm 3.1$ & $+3.10 \pm 0.40$ \\
\hline T. infestans & Females & 27 & $23.5 \pm 5.1$ & $22.6 \pm 5.3$ & $-1.00 \pm 0.20$ \\
\hline
\end{tabular}

No: number of insects; SD: standard deviation

Table 5 Mean quantity of blood ingested (mg) for each instar of Triatoma infestans, T. sordida and T. guasayana.

\begin{tabular}{|c|c|c|c|c|c|c|c|}
\hline Vector & Instar & $\mathrm{Nr}$ & Min. & Max. & Mean \pm DS & $\mathrm{F}$ & $\mathrm{P}<$ \\
\hline T. sordida & II & 31 & 3.50 & 6.0 & $5 \pm 1 x$ & 60.90 & 0.001 \\
\hline T. guasayana & II & 32 & 4.10 & 24.5 & $14 \pm 11^{1}$ & & \\
\hline T. infestans & II & 36 & 17.6 & 44.4 & $32 \pm 13$ & & \\
\hline T. sordida & III & 39 & 9.80 & 21.9 & $16 \pm 6 x$ & 58.40 & 0.001 \\
\hline T. guasayana & III & 44 & 13.50 & 25.1 & $20 \pm 6 x^{1}$ & & \\
\hline T. infestans & III & 39 & 23.0 & 72.0 & $50 \pm 25$ & & \\
\hline T. sordida & IV & 44 & 28.0 & 55.0 & $40 \pm 13$ & 57.40 & 0.001 \\
\hline T. guasayana & IV & 44 & 18.0 & 55.0 & $40 \pm 20^{1}$ & & \\
\hline T. infestans & IV & 44 & 56.0 & 90.0 & $74 \pm 17$ & & \\
\hline T. sordida & V & 61 & 53.0 & 140.0 & $100 \pm 45$ & 12.40 & 0.001 \\
\hline T. guasayana & V & 64 & 68.0 & 170.0 & $115 \pm 51^{1}$ & & \\
\hline T. infestans & V & 64 & 107.0 & 176.0 & $139 \pm 34$ & & \\
\hline T. sordida & Males & 17 & 51.0 & 73.0 & $64 \pm 11$ & 127.90 & 0.001 \\
\hline T. guasayana & Males & 26 & 31.0 & 71.0 & $53 \pm 20^{1}$ & & \\
\hline T. infestans & Males & 25 & 103.0 & 135.0 & $124 \pm 16$ & & \\
\hline T. sordida & Females & 23 & 55.0 & 88.0 & $71 \pm 14$ & 281.70 & 0.001 \\
\hline T. guasayana & Females & 26 & 30.0 & 70.0 & $48 \pm 19^{1}$ & & \\
\hline T. infestans & Females & 27 & 130.0 & 165.0 & $146 \pm 14$ & & \\
\hline
\end{tabular}

No: number of insects; SD: standard deviation; ${ }^{1}$ test comparison of the mean: DM: reduced deviation $>30 ; \mathrm{F}=$ student test $>30$ 
Table 6 Mean number of different forms found in triatomine feces of Trypanosoma cruzi (metacyclic-trypomastigotes, epimastigotes and other forms) in feces of triatomine instars (mean for vectors after examination at 100x to quantify the number of parasites in 200 fields).

\begin{tabular}{|c|c|c|c|c|c|c|c|c|c|c|c|c|c|}
\hline \multirow{2}{*}{ Instar } & \multirow{2}{*}{ No } & \multicolumn{3}{|c|}{ Metacyclicstrypomastigotes } & \multicolumn{3}{|c|}{ Epimastigotes } & \multicolumn{3}{|c|}{ Other forms } & \multicolumn{3}{|c|}{ Total forms } \\
\hline & & Min. & Max. & Mean \pm DS & Min. & Max. & Mean \pm DS & Min. & Max. & Mean \pm DS & Min. & Max. & Mean \pm DS \\
\hline \multicolumn{14}{|c|}{ T. guasayana } \\
\hline Males & 5 & 7.6 & 16.0 & $12.2 \pm 2.8$ & 88.9 & 113.7 & $100.4 \pm 10.2$ & 14.7 & 20.6 & $18.4 \pm 3.0$ & 111.2 & 150.3 & $131.0 \pm 13.0$ \\
\hline Females & 5 & 9.5 & 13.5 & $10.8 \pm 1.3$ & 90.6 & 125.3 & $108.8 \pm 14.3$ & 9.9 & 14.9 & $13.4 \pm 2.3$ & 110.0 & 153.7 & $133.0 \pm 16.8$ \\
\hline $\mathrm{V}$ & 10 & 10.9 & 18.0 & $15.2 \pm 3.4$ & 102.5 & 153.7 & $128.6 \pm 27.3$ & 12.9 & 19.7 & $18.4 \pm 4.2$ & 126.3 & 191.4 & $162.2 \pm 33.6$ \\
\hline IV & 10 & 14.8 & 25.9 & $21.0 \pm 5.9$ & 125.2 & 220.6 & $174.1 \pm 43.3$ & 19.5 & 34.4 & $28.8 \pm 8.3$ & 159.5 & 280.9 & $223.9 \pm 53.4$ \\
\hline III & 10 & 8.7 & 18.9 & $13.9 \pm 4.5$ & 89.2 & 147.6 & $122.1 \pm 29.7$ & 12.9 & 19.9 & $18.3 \pm 3.6$ & 110.8 & 186.4 & $154.3 \pm 36.0$ \\
\hline Total & 40 & 9.4 & 21.0 & $15.4 \pm 5.4$ & 89.4 & 165.6 & $132.4 \pm 39.0$ & 12.6 & 25.8 & $20.4 \pm 7.2$ & 111.4 & 212.4 & $168.1 \pm 49.6$ \\
\hline \multicolumn{14}{|c|}{ T. sordida } \\
\hline Males & 4 & 5.8 & 15.9 & $11.5 \pm 5.2$ & 70.6 & 128.7 & $104.8 \pm 31.4$ & 30.6 & 51.7 & $45.0 \pm 12.5$ & 107.0 & 196.3 & $161.3 \pm 44.4$ \\
\hline Females & 4 & 6.8 & 15.7 & $10.8 \pm 3.2$ & 69.7 & 91.4 & $86.5 \pm 8.8$ & 17.3 & 33.1 & $28.0 \pm 8.1$ & 93.8 & 140.2 & $125.3 \pm 10.5$ \\
\hline $\mathrm{V}$ & 10 & 9.8 & 15.6 & $13.5 \pm 2.9$ & 76.4 & 135.2 & $111.2 \pm 28.8$ & 13.4 & 25.2 & $21.3 \pm 5.9$ & 99.6 & 176.0 & $146.0 \pm 34.5$ \\
\hline IV & 11 & 8.5 & 21.9 & $16.5 \pm 6.6$ & 76.5 & 148.2 & $120.5 \pm 36.2$ & 8.3 & 30.3 & $21.5 \pm 11.1$ & 93.3 & 200.4 & $158.5 \pm 49.9$ \\
\hline III & 10 & 5.8 & 18.9 & $12.4 \pm 7.5$ & 68.4 & 136.3 & $107.1 \pm 35.9$ & 7.6 & 26.2 & $18.7 \pm 9.8$ & 81.8 & 181.4 & $138.2 \pm 50.6$ \\
\hline Total & 39 & 8.2 & 20.3 & $13.6 \pm 5.8$ & 69.3 & 136.1 & $109.6 \pm 32.1$ & 10.7 & 33.6 & $23.8 \pm 11.8$ & 88.2 & 190.0 & $146.9 \pm 42.9$ \\
\hline \multicolumn{14}{|c|}{ T. infestans } \\
\hline Males & 6 & 15.7 & 28.8 & $23.3 \pm 6.5$ & 88.4 & 210.9 & $157.8 \pm 64.5$ & 15.8 & 34.2 & $26.0 \pm 9.3$ & 120.2 & 273.9 & $207.2 \pm 73.0$ \\
\hline Females & 5 & 14.9 & 28.5 & $21.6 \pm 5.9$ & 107.4 & 159.8 & $144.2 \pm 24.3$ & 18.5 & 34.7 & $29.8 \pm 8.7$ & 140.8 & 223.0 & $195.6 \pm 19.4$ \\
\hline $\mathrm{V}$ & 10 & 12.8 & 25.5 & $20.2 \pm 6.5$ & 69.3 & 149.7 & $119.3 \pm 40.7$ & 9.8 & 28.7 & $20.3 \pm 9.7$ & 91.9 & 203.9 & $159.8 \pm 50.9$ \\
\hline IV & 5 & 18.9 & 27.3 & $23.8 \pm 4.4$ & 88.4 & 209.7 & $158.2 \pm 58.3$ & 25.2 & 39.6 & $36.0 \pm 7.3$ & 132.5 & 276.6 & $218.0 \pm 59.7$ \\
\hline III & 7 & 10.5 & 19.1 & $15.6 \pm 4.2$ & 78.9 & 111.6 & $102.6 \pm 14.9$ & 10.6 & 27.9 & $21.3 \pm 9.0$ & 100.0 & 158.6 & $139.4 \pm 17.2$ \\
\hline Total & 33 & 13.8 & 25.6 & $20.5 \pm 6.1$ & 74.9 & 168.3 & $132.4 \pm 46.4$ & 13.6 & 33.4 & $25.4 \pm 10.2$ & 102.3 & 227.3 & $178.3 \pm 54.7$ \\
\hline
\end{tabular}

No: number of insects; SD: standard deviation

(Schofield et al 1991). The low mean weight of T. sordida adults allows them to easily disperse and at the same time create new colonies (Crocco \& Catalá 1996).

The results obtained in this study support the statements made by some authors. Triatoma sordida needs average blood feeding of 5, 16, 40, $100 \mathrm{mg}$ of blood for nymphal stages: $\mathrm{N} 2, \mathrm{~N} 3, \mathrm{~N} 4, \mathrm{~N} 5$ respectively, and $64 \mathrm{mg}$ and $71 \mathrm{mg}$ for adult males and females respectively, in order to defecate during feeding or after finishing it. Whereas for T. guasayana 14 , 20,40,115 mg of blood for nymphal stages: N2, N3, N4, $\mathrm{N} 5$, and $53 \mathrm{mg}$ and $48 \mathrm{mg}$ for male and females adults are needed respectively.

The results of the attacking time, feeding time and defecating time demonstrated the superiority of $T$. infestans since it started feeding, ingested blood and defecated faster than the other species. Although T. sordida is faster than $T$. guasayana in attacking time, its feeding time is prolonged in the first stages. Its blood ingestion is frequently incomplete due to interruptions and the insect will not start to defecate. Crocco \& Catalá (1996) observed in T. infestans and $T$. sordida, that the first species was the most efficient except for the females. Our data do not show any differences regarding sex, but the authors used another index (Zeledon et al 1977) without considering the excretions emitted before the end of feeding. However, we agree that the fastest defecation occurs in the first nymphal instars (2 to 4$)$.

Of all the Triatominae of the genus Triatoma, T. infestans seems to be the species which produces the highest percentage of trypomastigotes. However, the metacyclogenesis of $T$. cruzi would be more efficient in the genera Rhodnius and Panstrongylus (Perlowagora-Scumlewicz \& Carvalho Moreira 1994), and from a comparative study of the differentiation of $T$. cruzi in various species of triatomines, these authors concluded that there was a direct relationship between Triatominae species and metacyclogenesis. Our data showed a higher production of metacyclic forms in T. infestans compared to T. sordida and $T$. guasayana, and we did not observe any differences between the different instars of the same species. Various intrinsic factors, such as hemolytic factors and lecithins, may affect the development of strains of $T$. cruzi and explain the differences observed in metacyclogenesis among species (Garcia \& Azumbuja 1991).

Of the three species studied, $T$. infestans has, without a doubt, the biggest vectorial capacity in the laboratory, and this is fully confirmed in the field where this species constitutes the main vector of Chagas disease in the Southern Cone countries 
(Schofield 1994). Triatoma sordida is in a more advanced domiciliary process (Noireau et al 1997), and this species is superior to $T$. guasayana regarding the feeding-defecation interval. Both species have very similar characteristics of metacyclogenesis, but this is insufficient for $T$. sordida to be considered an efficient vector in infested housing and its population dynamics may explain its low vectorial capacity (Noireau et al 1997). This last parameter, which was not considered in this study, should be studied in T. guasayana to see if it can be considered as a possible vector.

In the wild, $T$. sordida and $T$. guasayana are species which occupy different peridomiciliary areas without coexisting, except in the "monte chaqueño" of Santiago del Estero, Argentina (Gajate et al 1996, Cecere et al 1999). Studies in Argentina on their dispersal capacity show that $T$. guasayana actively invades not only the peridomiciliary area but also the domestic area, where it is numerically superior to $T$. sordida. However, none of these species has been able to colonize houses, probably because during the stage of domiciliary re-infestation, they have to compete with $T$. infestans which has a greater capacity for building important colonies within the same area (Carcavallo et al 1988, Wisnivesky-Colli et al 1993, Cecere et al 1999, Noireau et al 1999).

Since $T$. guasayana was found infected with $T$. cruzi in the wild, as well as invading housing, although without colonizing it, various authors support the hypothesis that this species would not only be acting as a link between the three cycles of parasite transmission (wild - peridomiciliary - domiciliary), but is also behaving as a possible secondary vector of the parasite in Argentina and is a potential substitute of $T$. infestans during the surveillance stage (Wisnivesky-Colli et al 1993, Gajate et al 1996, Cecere et al 1999).

Although the need for more studies on the vectorial capacity of $T$. sordida and T. guasayana is recognized, the results obtained in this study show that $5^{\text {th }}$ instar nymphs would be capable of transmitting $T$. cruzi, due to characteristics of the vectorial capacity of these species which show that they begin ingesting blood and feed and defecate quickly.

\section{Acknowledgments}

To the Tropical Diseases Laboratory of I.B.B.A. for their kind cooperation; the Phytochemistry Department for supplying the T. cruzi strain; the Insectarium for providing the Triatomine species studied; and all the people who helped me directly or indirectly in this project. Also Dr. Eddy Martinez for their constructive criticism.

\section{References}

Bar M E Oscherov E B, Damborsky M P (1993) Presencia de Triatoma sordida Stal, 1859 en ecotopos urbanos de la ciudad de Corrientes, Argentina. Rev Saúde Públ 27:117-122.

Barretto M P (1971) Estudos sôbre reservatórios e vectores silvestres do Trypanosoma cruzi. XLV: inquérito preliminar sôbre triatomíneos silvestres no Sul do estado de Mato Grosso, Brasil (Hemiptera: Reduviidae). Rev Bras Biol 31: 225-233.

Bérenger J M, Blanchet D (2007) A new species of the genus Panstrongylus from French Guiana (Heteroptera; Reduviidae; Triatominae). Mem Inst Oswaldo Cruz 102: 733-736.

Canale D M (2000) Actualización de la biología de triatominos. Medicina 6 (supl III): 14-15.

Carcavallo R U, Canale D, Martínez A (1988) Hábitat de triatomineos argentinos y zonas ecológicas donde prevalecen. Chagas, Buenos Aires, 5: 8-17.

Carcavallo R U, Martínez A (1985) Biología, ecología y distribución geográfica de los Triatominos americanos, p.149-208. En Carcavallo R U, Rabinovich J E, Tonn R J (eds) Factores biológicos y ecológicos en la enfermedad de Chagas. Ministerio de Salud y Acción Social, Buenos Aires, Argentina, Tomo I, $250 \mathrm{p}$.

Cecere M C, Castañera M B, Canale D M, Chuit R, Gurtler R E (1999) Trypanosoma cruzi infection in Triatoma infestans and other triatomines: long-term effects of a control program in rural northwestern Argentina. Rev Panam Salud Publ 5: 392- 399.

Costa J M, Argolo A M, Felix, M (2006) Redescription of Triatoma melanica Neiva \& Lent, (1941, new status (Hemiptera: Reduviidae: Triatominae). Zootaxa 1385: 47-52.

Costa J M, Felix M (2007) Triatoma juazeirensis sp. nov. from the state of Bahia, Northeastern Brazil (Hemiptera: Reduviidae: Triatominae). Mem Inst Oswaldo Cruz 102: 87-90.

Crocco L B, Catalá S (1996) Feeding and defecation patterns in Triatoma sordida. Mem Inst Oswaldo Cruz 91: 409-413.

Días J C P (1988) Controle de vetores da doença de Chagas no Brasil e risco de reinvasão domiciliar por vetores secundários. Mem Inst Oswaldo Cruz 83: 387-39.

Diotaiuti L, Loyola C F, Falcão P L, Dias J C P (1993) The ecology of Triatoma sordida in natural environments in two different regions of the state of Minas Gerais, Brazil. Rev Inst Med Trop 35: 237-245.

Ekkens D B (1981) Nocturnal flights of Triatoma (Hemiptera: Reduviidae) in Sabino Canyon, Arizona. J Med Entomol 18: 211-227.

Forattini O P (1980) Biogeografía, origem e distribuição da domiciliação de triatomíneos no Brasil. Rev Saúde Públ 14: 265-299.

Forattini O P, Barata J M S, Santos J F, Silveira A C (1982) Hábitos alimentares, infecção de triatomineos domiciliados na Região Central do Brasil. Rev Saúde Pública 16: 171-204.

Forattini O P, Ferreira O A, Rocha e Silva E O, Rabello E X, Santos J L F (1971) Aspectos ecológicos da tripanossomíase americana. II. Distribuição e dispersão local de triatomíneos em ecótopos naturais e artificiais. Rev Saúde Públ 5: 163-191.

Gajate P P, Bottazzi M V, Pietrokovsky S M, Wisnivesky-Colli C (1996) Potential colonization of the peridomicilie by Triatoma guasayana (Hemiptera: Reduviidae) in Santiago del Estero, Argentina. J Med Entomol 33: 635-639. 
Galvão C, Ângulo V M (2006) Belminus corredori, a new species of Bolboderini (Hemiptera: Reduviidae: Triatominae) from Santander, Colombia. Zootaxa 1241: 61-68.

Galvão C, Carcavallo R U, Rocha D S, Jurberg J (2003) A checklist of the current valid species of the subfamily Triatominae Jeannel 1919 (Hemiptera, Reduviidae) and their geographical distribution with nomenclatural and taxonomic notes. Zootaxa 202: $1-36$

Garcia E, Azambuja P (1991) Development and interactions of Trypanosoma cruzi within the insect vector. Parasitol Today 7: $240-244$.

Gürtler R E, Cécere M C, Lauricella M A, Petersen R M, Chuit R, Segura E L, Cohen J E (2005) Incidence of Trypanosoma cruzi infection among children following domestic reinfestation after insecticide spraying in rural northwestern Argentina. Am J Trop Med and Hyg 73: 95-103.

Juarez E Castro, Silva E P (1982) Comportamento do Triatoma sordida em condições de laboratorio. Rev Saúde Publica 16: $1-36$.

Kirk M L, Schofield C J (1987) Density-dependent timing of defecation by Rhodnius prolixus, and its implications for the transmission of Trypanosome cruzi. Trans R Soc Trop Med Hyg 81: 348-349.

Lehane M J, Schofield C J (1982) Flight initiation in Triatoma infestans (Klug) (Hemiptera: Reduviidae). Bul Entomol Res 72: 497-510.

Lent H, Wygodzinsky P (1979) Revision of the Triatominae (Hemiptera, Reduviidae) and their significance as vectors of Chagas'disease. Bull Amer Mus Nat Hist 163: 125-520.

Martinez E Chávez T, Sossa D, Aranda R, Vargas B, Vidaurre P (2007) Triatoma boliviana sp. n. de los valles subandinos de La Paz, Bolivia (Hemiptera: Reduviidae: Triatominae), similar a Triatoma nigromaculata Stål, 1859. Bol Inst Invest Salud Desar 3: 1-11.

McEwen P K, Lehane M J (1993) Factors influencing flight initiation in the triatomine bug Triatoma sordida (Hemiptera: Reduviidae). Insect Sc Appl 14: 461- 464.

Mello D A (1980) Algunos aspectos de hábitos alimenticios en condiciones de laboratorio de Triatoma sordida y Rhodnius neglectus. Rev Bras Biol 40: 323- 326.

Noireau F, Brenière F, Ordoñez J, Cardozo L, Morochi W, Gutiérrez T, Bosseno M F, García S, Vargas F, Yaksic N, Dujardin J P, Peredo C, Wisnivesky-Colli C (1997) Low probability of transmission of Trypanosoma cruzi to man by domestic Triatoma sordida in Santa Cruz Department, Bolivia. Trans R Soc Trop Med Hyg 91: 653-656.

Noireau F, Flores R, Gutierrez T, Abad-Franch F, Flores E, Vargas F (2000) Natural ecotopes of Triatoma infestans dark morph and other wild triatomines in the Bolivian Chaco. Trans R Soc Trop Med Hyg 94: 23-27.

Noireau F, Flores R, Gutierrez T, Vargas F, Brenière F, Dujardin J P (1999) Triatominae en Bolivia: importancia actual de los candidatos vectores, p.127-133. In Alfred Cassab J, Noireau F,
Guillén G La enfermedad de Chagas en Bolivia - conocimientos científicos al inicio del Programa de Control (1998-2002). Ministerio de Salud y Previsión Social, OMS/OPS, IRD, IBBA eds, La Paz, 355p.

Noireau F, Gutierrez T, Flores R, Brenière F, Bosseno MF, Wisnivesky-Colli C (1998) Ecogenetics of Triatoma sordida and T. guasayana (Hemiptera: Reduviidae) in the Bolivian Chaco. Mem Inst Oswaldo Cruz 94: 451-457.

OMS (1991) Control de la enfermedad de Chagas. Inf Téc No. 811.

OMS (2007) Reporte del grupo de trabajo científico sobre la enfermedad de Chagas. 17 al 20 de abril 2005. Actualizado Julio 2007. TDR/GTC/06. Buenos Aires, Argentina.

Perlowagora-Szumlewicz A (1973) Species and stage interaction in the feeding behavior of vectors of Chagas'disease. Rev Inst Med Trop 15: 139-150.

Perlowagora-Scumlewicz A, Carvalho Moreira C J (1994) In vivo differentiation of Trypanosoma cruzi-1. Experimental evidence of the influence of vector species on metacyclogenesis. Mem Inst Oswaldo Cruz 89: 603-618.

Rojas-de-Arias A (1996) Chagas' disease in Paraguay. Washington, DC, Pan-American Health Organization, World Health Organization, 280p.

Rosa R, Salvatella R (1995) Diagnóstico de situación epidemiológica actual de la enfermedad de Chagas en Uruguay. Bol Soc Zool Uruguay 9:6-11.

Ruas Neto A L, Krug L (1995) Epidemiologia da doença de Chagas no Rio Grande do Sul: A distribução vetorial. Bol Soc Zool Uruguay 9: 20-32.

Sandoval C M, Pabón E, Jurberg J, Galvão C (2007) Belminus ferroae n.sp. from the Colombian north-east, with a key to the species of the genus (Hemiptera: Reduviidae: Triatominae). Zootaxa 1443: 55-64.

Schofield C J (1991) Biosystematics of the Triatominae, p.284312. In Service M W (ed) Biosystematics of haematophagous insect. Systemetics Association, special vol 37, Clarendon Press, Oxford, 370p.

Schofield C J (1994) Triatominae: biology and control. Eurocommunica Publications, West Sussex, 80p.

Schofield C J, Jannin J, Salvatella R (2006) The future of Chagas disease control. Trends Parasitol 22: 583-588.

Sjogren R D, Ryckman R E (1996) Epizootiology of Trypanosoma cruzi in southwestern North America. Part VIII. Nocturnal flights of Triatoma proctata (Uhler) as indicated by collections in black light traps (Hemiptera, Reduviidae, Triatominae). J Med Entomol 3: 81-92.

Trumper E V, Gorla D E (1991) Density - dependent timing of defecation by Triatoma infestans. Trans R Soc Trop Med Hyg 85: 800-802.

Usinger R L, Wygodzinsky P, Ryckman R E (1966) The biosystematics of Triatominae. Ann Rev Entomol 11: 309330. 
Vezzani D, Schweigmann N, Pietrokovsky S M, Wisnivesky- Colli C (2001) Characterization of Triatoma guasayana biotopes in a hardwood forest of Santiago del Estero, Argentina. Mem Inst Oswaldo Cruz 95: 459- 466.

Wisnivesky-Colli C, Gürtler R E, Solarz N D, Schweigmann N J, Pietrokovsky S M, Alberti A, Flo J (1993) Dispersive flight and house invasion by Triatoma guasayana and Triatoma sordida in Argentina. Mem Inst Oswaldo Cruz 88: 27-32.
Zeledón R, Alvarado R, Jiron L F (1977) Observations of the feeding and defecation of three Triatominae species (Hemiptera, Reduviidae). Acta Trop 34: 65-77.

Received 26/XII/06. Accepted 22/VI/10. 